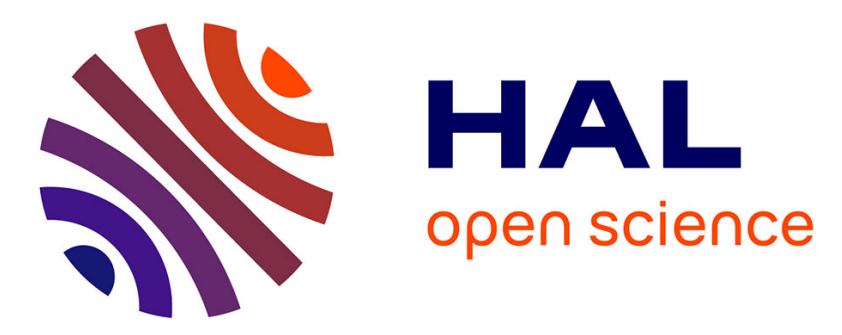

\title{
Dispersion curve veering of longitudinal guided waves propagating inside prestressed seven-wire strands
}

Fabien Treyssede

\section{To cite this version:}

Fabien Treyssede. Dispersion curve veering of longitudinal guided waves propagating inside prestressed seven-wire strands. Journal of Sound and Vibration, 2016, 367, pp.56-68. 10.1016/j.jsv.2015.12.050 . hal-01346127

\author{
HAL Id: hal-01346127 \\ https://hal.science/hal-01346127
}

Submitted on 18 Jul 2016

HAL is a multi-disciplinary open access archive for the deposit and dissemination of scientific research documents, whether they are published or not. The documents may come from teaching and research institutions in France or abroad, or from public or private research centers.
L'archive ouverte pluridisciplinaire HAL, est destinée au dépôt et à la diffusion de documents scientifiques de niveau recherche, publiés ou non, émanant des établissements d'enseignement et de recherche français ou étrangers, des laboratoires publics ou privés. 


\title{
Dispersion curve veering of longitudinal guided waves propagating inside prestressed seven-wire strands
}

\author{
Fabien Treyssède ${ }^{\mathrm{a}, *}$ \\ ${ }^{a}$ LUNAM Université, IFSTTAR, GERS, GeoEND, F-44340 Bouguenais, France
}

\begin{abstract}
Elastic guided waves are of interest for the non destructive evaluation of cables. Such structures are usually helical, multi-wired and highly prestressed, which greatly complicates the understanding of wave propagation from a theoretical point of view. A remarkable feature is the occurence of a missing frequency band in experimental time-frequency diagrams, sometimes referred to as notch frequency in the literature. The central frequency of this band increases under tensile loads. Recently, a numerical model has been proposed to compute the dispersion curves of prestressed helical seven-wire waveguides. Results have shown that the notch frequency indeed corresponds to a curve veering phenomenon between two longitudinal-like modes and that the increase of the notch under tensile loads is mainly due to interwire contact mechanisms. The main goal of this paper is to highlight the origin of this curve veering phenomenon, which is still unexplained up to the author's knowledge. This paper also provides further results which allow to clarify the accuracy of numerical solutions as well as the influence of contact assumptions. First, the static part of the model, necessary to compute the prestress state including contact effects, is checked from reference analytical solutions. Owing to the importance of contact, the accuracy of results is discussed both in statics and in dynamics. The influence of slip contact conditions is outlined. Then, some numerical tests are conducted by varying the Poisson coefficient and the helix lay angle. These tests allows to find out that the radial displacement constraint imposed on peripheral wires by the central one in the contact regions constitutes the main source of curve veering. More precisely, it is shown that a similar curve veering does occur for an uncoupled single peripheral wire when constrained by a radially blocked motion localised in its contact zone. Indeed, such a localised boundary condition completely breaks the circular symmetry of the wire cross-section, yielding coupling between longitudinal, flexural and torsional motion together with curve veering phenomena.
\end{abstract}

Keywords: wave, prestress, cable, strand, contact, curve veering

\section{Introduction}

Cables are widely used in modern constructions. In order to assess their structural health, the development of non destructive evaluation techniques is necessary. Guided wave based methods are of particular interest because these waves can propagate over long distances, hereby increasing the potential distance of inspection. However, the understanding of mechanisms governing the propagation of guided waves is particularly complicated owing to the helical and multi-wire structure of strands, the basic elements constituting cables. The complexity of the problem is further increased by the presence of high tensioning forces applied on cables. These huge axial loads can influence the propagation of waves.

There exists a wide variety of strands. A common type, widely encountered in modern bridges, is the so-called seven-wire strand, made by one central cylindrical wire surrounded by six helical peripheral ones

\footnotetext{
* Corresponding author

URL: fabien.treyssede@ifsttar.fr (Fabien Treyssède)
} 


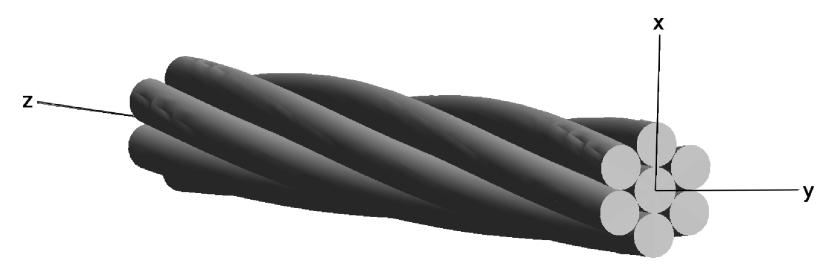

Figure 1: Geometry of a seven-wire strand.

as sketched by Fig. 1. Experiments have shown that a seven-wire strand cannot generally be modeled as an effective cylinder $[1,2,3]$. More complex models of waveguides are hence needed. Recently, numerical models have been proposed in order to subsequently account for: the helical geometry of a single wire $[4,5]$, the interwire coupling [6] and the effect of axial loads [7, 8]. These models are based on a semi-analytical finite element (SAFE) method. Good agreement with experiments have been found on the so-called notch frequency and its increase under applied tensile loads. The notch frequency represents a missing frequency band experimentally observed in time-frequency diagrams [1,2] and is typical of strands. In Ref. [6], it has been shown that the notch frequency indeed corresponds to a curve veering phenomenon between the dispersion curves of two distinct longitudinal-like wave modes. Curve veering phenomena are generally found in eigenvalue problems of weakly coupled systems $[9,10]$ and can be defined as the repulsion of two modal branches, veering away from each other instead of crossing. In Ref. [8], numerical tests have demonstrated that the noth frequency increase under tensile loads is indeed mainly influenced by interwire contact.

Nevertheless, some modeling works are still required in order to improve the model and the understanding of wave propagation inside strands. The modeling of contact needs to be checked with reference solutions and the origin of the curve veering phenomenon has not been explained yet. Besides, the convergence of numerical results have to be assessed, as it is well known in contact problems that a sufficiently fine mesh is required in contact regions to achieve an accurate modeling. The present paper aims to highlight these issues.

\section{Theoretical background}

\subsection{SAFE modeling of helical waveguides under prestress}

The SAFE method has been widely considered in Cartesian coordinates, for the analysis of straight waveguides (see for instance Refs. [11, 12]). It has also been proposed in cylindrical coordinates for the study of toroidal waveguides $[13,14]$.

The analysis of guided waves inside helical strands requires a specific curvilinear coordinate system, called twisting coordinate system. Such a system has constant non zero torsion but zero curvature, and hence corresponds to a particular case of helical system. This coordinate system has been used for pretwisted beams [15]. Under the assumption of a linear elastic material, isotropic and homogeneous, it has been shown in Ref. [6] that a twisting system allows to preserve translation invariance in seven-wire strands, which hereby yields a theoretical proof for the existence of guided waves in such structures. With this kind of system, the cross-section plane remains perpendicular to the straight axis but rotates around this axis by following peripheral wires. Since the central wire is circular and isotropic, the cross-section of the whole structure and its material properties remain translationally invariant in a twisting system. The torsion of the twisting system is given by $\tau_{0}=2 \pi / L_{0}$, with $L_{0}$ denoting the helix pitch of peripheral wires under prestress. This section briefly reviews the main equations of the SAFE method written in a twisting system and including prestress effects. Further details can be found in Refs. [7, 8].

Let us denote $z$ the straight axis of the waveguide, fixed to the Cartesian system, $(x, y)$ the crosssection twisting coordinates, $k$ the axial wavenumber and $\omega$ the angular frequency. The application of a SAFE method consists in assuming an $\mathrm{e}^{\mathrm{i}(k z-\omega t)}$ dependence of acoustic fields before finite element (FE) discretization. Therefore, only the two-dimensional cross-section into the $(x, y)$ plane of the structure has to be meshed. One points out that the $\mathrm{e}^{\mathrm{i} k z}$ field dependence implies that axial variables must be separable 
from transverse variables in the governing equations of motion (this separation of variable is actually possible thanks to the proof of translational invariance along the $z$-axis that holds in a twisting system $[6,16])$.

The application of a SAFE method leads to the following matrix system governing wave propagation inside prestressed strands:

$$
\left\{\mathbf{K}_{1 \sigma}-\omega^{2} \mathbf{M}+\mathrm{i} k\left(\mathbf{K}_{2 \sigma}-\mathbf{K}_{2 \sigma}^{\mathrm{T}}\right)+k^{2} \mathbf{K}_{3 \sigma}\right\} \mathbf{U}=\mathbf{0},
$$

with the element matrices:

$$
\begin{array}{r}
\mathbf{M}^{e}=\int_{S_{0}^{e}} \rho_{0} \mathbf{N}^{e \mathrm{~T}} \mathbf{N}^{e} \mathrm{~d} x \mathrm{~d} y, \\
\mathbf{K}_{1 \sigma}^{e}=\mathbf{K}_{1}^{e}+\int_{S_{0}^{e}} \mathbf{N}^{e \mathrm{~T}} \mathbf{G}_{x y}^{\mathrm{T}} \boldsymbol{\Sigma}_{0} \mathbf{G}_{x y} \mathbf{N}^{e} \mathrm{~d} x \mathrm{~d} y \\
\mathbf{K}_{2 \sigma}^{e}=\mathbf{K}_{2}^{e}+\int_{S_{0}^{e}} \mathbf{N}^{e \mathrm{~T}} \mathbf{G}_{x y}^{\mathrm{T}} \boldsymbol{\Sigma}_{0} \mathbf{G}_{z} \mathbf{N}^{e} \mathrm{~d} x \mathrm{~d} y, \\
\mathbf{K}_{3 \sigma}^{e}=\mathbf{K}_{3}^{e}+\int_{S_{0}^{e}} \mathbf{N}^{e \mathrm{~T}} \mathbf{G}_{z}^{\mathrm{T}} \boldsymbol{\Sigma}_{0} \mathbf{G}_{z} \mathbf{N}^{e} \mathrm{~d} x \mathrm{~d} y, \\
\mathbf{K}_{1}^{e}=\int_{S_{0}^{e}} \mathbf{N}^{e \mathrm{~T}} \mathbf{L}_{x y}^{\mathrm{T}} \mathbf{C}_{0} \mathbf{L}_{x y} \mathbf{N}^{e} \mathrm{~d} x \mathrm{~d} y, \\
\mathbf{K}_{2}^{e}=\int_{S_{0}^{e}} \mathbf{N}^{e \mathrm{~T}} \mathbf{L}_{x y}^{\mathrm{T}} \mathbf{C}_{0} \mathbf{L}_{z} \mathbf{N}^{e} \mathrm{~d} x \mathrm{~d} y, \\
\mathbf{K}_{3}^{e}=\int_{S_{0}^{e}} \mathbf{N}^{e \mathrm{~T}} \mathbf{L}_{z}^{\mathrm{T}} \mathbf{C}_{0} \mathbf{L}_{z} \mathbf{N}^{e} \mathrm{~d} x \mathrm{~d} y .
\end{array}
$$

where $\rho_{0}$ and $\mathbf{C}_{0}$ denote the mass density and the matrix of elastic properties. On one two-dimensional element $e$, the finite element approximation of the displacement vector $\mathbf{u}$ is given by: $\mathbf{u}(x, y)=\mathbf{N}^{e}(x, y) \mathbf{U}^{e}$, where $\mathbf{N}^{e}$ is the matrix of the shape functions and $\mathbf{U}^{e}$ is the vector of nodal displacements, with 3 degrees of freedom per node, written in the orthonormal Serret-Frenet basis associated with the twisting system. The matrix $\boldsymbol{\Sigma}_{0}$ depends on $\boldsymbol{\sigma}_{0}$, the Cauchy prestress (i.e. the stress tensor associated with the static prestress state). The operators $\mathbf{L}_{x y}$ and $\mathbf{G}_{x y}$ depend on the torsion $\tau_{0}$. The expressions of $\mathbf{\Sigma}_{0}, \mathbf{L}_{x y}, \mathbf{L}_{z}, \mathbf{G}_{x y}$ and $\mathbf{G}_{z}$ are recalled in Appendix A.

Equations (1) and (2) can be viewed as the so-called linearized updated Lagrangian formulation of nonlinear mechanics (see e.g. [17] for instance), here extended to twisting coordinates and adapted to a SAFE formulation. The subscript 0 refers to the statically prestress configuration. It is noteworthy that the element matrices must be expressed on the prestressed cross-section $S_{0}$, statically deformed by the axial load. In practice, this means that the initial FE mesh should be updated to the prestressed geometry before the computation of SAFE matrices. Under the assumption of small strain, which is used in this paper, mechanical properties can be considered as independent of strain so that the following equalities hold: $\mathbf{C}_{0}=\mathbf{C}_{i}$ and $\rho_{0}=\rho_{i}$, where the subscript $i$ refers to the initial configuration (undeformed, unprestressed).

In Eqs. (2), the integral terms in $\boldsymbol{\Sigma}_{0}$ correspond to the so-called geometric stiffness, related to the presence of a prestress field $\left(\boldsymbol{\sigma}_{0} \neq 0\right)$. It has to be noticed that the prestressed state must not vary along the $z$-axis, otherwise the separation of the $z$-variable would not be possible. In practice, under axial loads applied at the end cross-sections of the helical structure, this condition is fulfilled far from the ends. Therefore, the static prestressed state can be considered as invariant along the axis.

\subsection{Static prestress state}

The first step of the analysis consists in computing the static prestress state of the seven-wire strand loaded by a constant tensile strain. One assumes that the influence of static non-linearities can be neglected on dynamics and the static prestress state will hence remain linear. Its computation can be achieved efficiently using a homogenization method specifically written in twisting coordinates. This allows to exploit the 
translational invariance property, and hereby, to restrict the problem to the cross-section. Homogenization splits the initial 3D elasticity problem into a 2D problem posed on the cross-section, as in a SAFE method, and a $1 \mathrm{D}$ straight beam problem. The overall beam behavior is computed thanks to the solution of the $2 \mathrm{D}$ problem, which also provides the local stress state.

The main FE formulation of the homogenization technique is briefly recalled. More technical details can be found in Ref. [7]. The prestressed state subjected to an axial strain can be determined by the following linear static computation:

$$
\mathbf{K}_{0} \mathbf{U}_{0}=\mathbf{F}_{0}
$$

with the element matrices:

$$
\mathbf{K}_{0}^{e}=\int_{S_{i}^{e}} \mathbf{N}^{e \mathrm{~T}} \mathbf{L}_{x y}^{\mathrm{T}} \mathbf{C}_{i} \mathbf{L}_{x y} \mathbf{N}^{e} \mathrm{~d} x \mathrm{~d} y, \mathbf{F}_{0}^{e}=-\int_{S_{i}^{e}} \mathbf{N}^{e \mathrm{~T}} \mathbf{L}_{x y}^{\mathrm{T}} \mathbf{C}_{i} \boldsymbol{\epsilon} \mathrm{d} x \mathrm{~d} y
$$

where $\epsilon=\left[\begin{array}{llllll}0 & 0 & \epsilon & 0 & 0 & 0\end{array}\right]^{\mathrm{T}}$ and $\epsilon$ is the prescribed axial strain. The rotational strain of the strand is set to zero. $\quad \mathbf{F}_{0}$ is hence an external load vector corresponding to an applied axial strain. $\mathbf{U}_{0}$ is the static nodal displacement vector. Note that $\mathbf{K}_{0}$ and $\mathbf{F}_{0}$ are integrated on $S_{i}$ (undeformed cross-section). In these expressions, the operator $\mathbf{L}_{x y}$ should be understood as the one given by Eq. (A.2) replacing $\tau_{0}$ with $\tau_{i}$, the torsion of the undeformed geometry. The torsions $\tau_{i}$ and $\tau_{0}$ are linked by: $\tau_{0}=\tau_{i} /(1+\epsilon)$.

The Cauchy prestress $\boldsymbol{\sigma}_{0}$, necessary for the calculation of SAFE matrices, can be post-processed from the solution of Eq. (3).

\section{Static contact modeling}

If Eq. (3) is solved in a unique step, the mechanics of contact is neglected. An iterative procedure is necessary to account for contact. In Ref. [7], it has been shown that the interwire contact significantly affects the value of the notch frequency. It is hence of importance to ensure that contact is properly modeled in statics prior to the analysis of wave propagation. The goal of this section is to refine the static model accounting for contact mechanics and to compare numerical results with analytical solutions in order to assess the accuracy of contact modeling.

\subsection{Analytical solution for two parallel cylinders}

Let us consider two parallel cylinders of radii $r_{c}$ and $r_{p}$ respectively (see Fig. 2). The material is the same for both cylinders. Following Hertz theory, the force per unit length $N_{0}$ normal to the contact surface is proportional to the indentation depth $d[18]$ :

$$
N_{0}=\frac{\pi}{8} \frac{E}{1-\nu^{2}} d
$$

where $E$ and $\nu$ denote Young's modulus and Poisson's ratio respectively. One recalls that Hertz theory assumes frictionless contact (zero friction coefficient).

The half contact width is given by $a=\sqrt{r^{\prime} d}$ where $1 / r^{\prime}=1 / r_{c}+1 / r_{p}$. As a consequence, the relation between the half contact width and the contact force is:

$$
a=\sqrt{\frac{8}{\pi} N_{0} \frac{1-\nu^{2}}{E} \frac{1}{\frac{1}{r_{c}}+\frac{1}{r_{p}}}}
$$

Despite the helical geometry of peripheral wires, the above formula indeed constitutes a good approximation to describe the contact width occurring between the central wire and the peripheral wires of a seven-wire strand, $r_{c}$ and $r_{p}$ denoting the radii of central and peripheral wires respectively. This will be shown by numerical results in Sec. 3.3. 


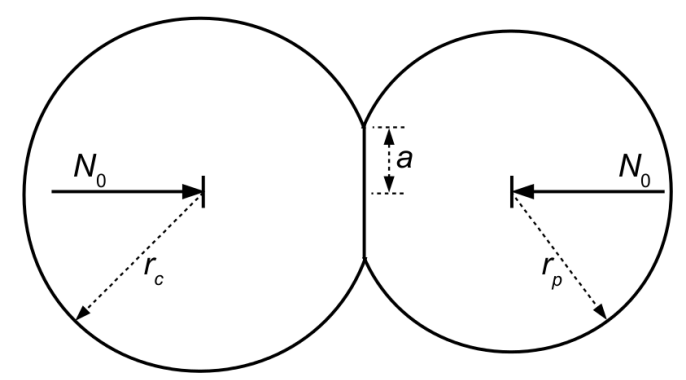

Figure 2: Hertz contact problem between two parallel cylinders.

\subsection{Numerical procedure}

The contact modeling of the tensile loading of a seven-wire strand can be simplified owing to the fact that small displacements can be assumed and no separation occurs during loading. The contact modeling approach used in this paper is on based on a node-to-node contact procedure together with a direct elimination method [19]. The FE mesh is refined near zones of potential contact. Owing to small deformations, matching meshes are used inside each interfacial zones where the central wire and each helical wire can possibly come into contact (see Fig. 3).

It is noteworthy that the diameter of peripheral wires (smaller than the central wire) is such that they do not contact each other. This is a widespread design criterion to minimize friction effects [20]. The gap between peripheral wires is indeed small and not visible in Fig. 3a. It remains nonzero in the loaded case also [8].

In order to account for contact, an iterative procedure must be used. For the $2 \mathrm{D}$ cross-section FE model used in this paper, the computation starts with single point contacts between wires. Then as the axial load is incremented, contact pairs of nodes are formed on both sides of the initial point-to-point contact zone. Each load increment is set manually from a visual inspection of the deformed FE mesh in order to check if the distance between pairs of nodes vanishes or not: if penetration occurs, the current increment is diminished otherwise it is increased, and the computation restarts from the current step. When the proper increment is found, the next incremental load step can start. The visual inspection of nodes in contact is performed on the updated cross-section (deformed geometry after the application of the axial load).

Once the gap between a pair of nodes is closed, the continuity of displacement is enforced at these nodes by the direct elimination method: the problem size is hence reduced by the degrees of freedom associated with one side of the contact interface. The displacement continuity at contact nodes is enforced along the three directions, which means that stick contact conditions are assumed (infinite friction). The effect of slip conditions (zero friction) will be briefly discussed later.

The iterative procedure can be summarized by the following linear incremental equation to solve for each incremental load step $j$ :

$$
\mathbf{K}_{0}^{j} \Delta \mathbf{U}=\frac{\Delta \epsilon}{\epsilon} \mathbf{P}^{j \mathrm{~T}} \mathbf{F}_{0}, \quad \mathbf{K}_{0}^{j}=\mathbf{P}^{j \mathrm{~T}} \mathbf{K}_{0} \mathbf{P}^{j}
$$

where $\mathbf{P}^{j}$ denotes the projection matrix which reduces the displacement degrees of freedom that are in contact at step $j$. The above projection preserves the properties of symmetry of matrices [19]. The total static displacement vector and the total prescribed strain are given by:

$$
\mathbf{U}_{0}=\sum_{j=1}^{J} \Delta \mathbf{U}, \epsilon=\sum_{j=1}^{J} \Delta \epsilon
$$

where $J$ is the number of increments dividing the total prescribed strain. The reaction force vector $\mathbf{R}_{0}$ can be computed from:

$$
\mathbf{R}_{0}=\mathbf{K}_{0} \mathbf{U}_{0}-\mathbf{F}_{0}
$$

For a given contact interface, the normal contact force $N_{0}$ is then obtained from the sum of contact nodal reactions of the interface projected onto its unit normal. 


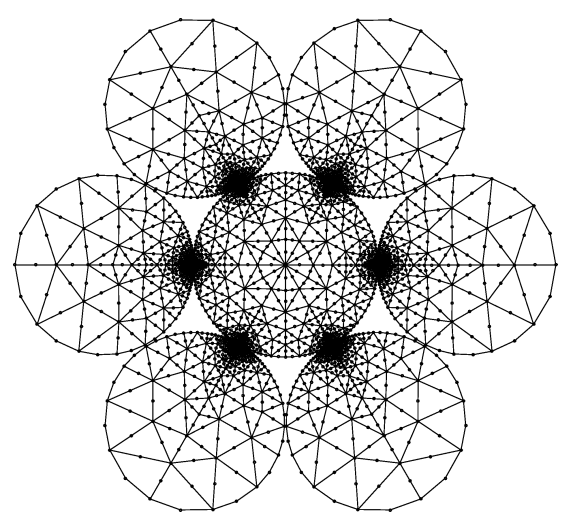

(a)

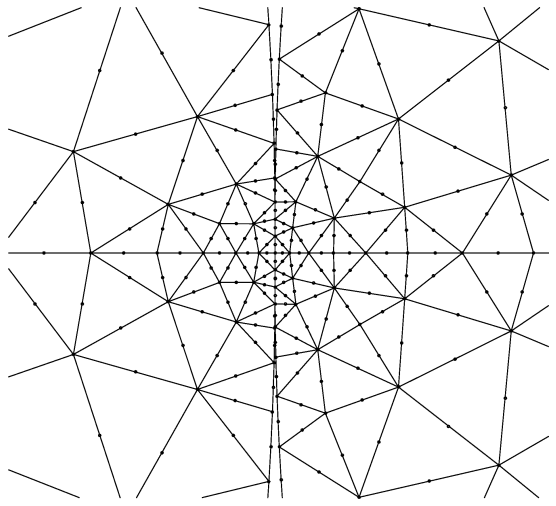

(b)

Figure 3: (a) Cross-section FE mesh of the seven-wire strand (12369 dofs), (b) zoom of the updated mesh for $\epsilon=0.6 \%$ in the contact region between the central wire and one peripheral wire.

\subsection{Results}

Throughout this paper, computations are performed for a seven-wire strand with the following parameters: $r_{p} / r_{c}=0.967, \phi=7.9^{\circ}, \nu=0.28$ and $\epsilon$ varying from 0 to $0.6 \%$. The helix lay angle $\phi$ is defined from the relation $\tan \phi=2 \pi\left(r_{c}+r_{p}\right) / L_{i}$ and the torsion of the twisting system is $\tau_{i}=2 \pi / L_{i}$, with $L_{i}$ denoting the length at rest of one helix pitch along the $z$-axis of peripheral wires. Normalized plots will be presented, but when specified, some results will be made dimensional with: $r_{c}=2.7 \mathrm{~mm}, E=2.17 \mathrm{e} 11 \mathrm{~Pa}, \rho=7800 \mathrm{~kg} / \mathrm{m}^{3}$ (material properties of steel).

The cross-section of the seven-wire strand has been meshed with Gmsh [21] and is shown in Fig. 3a. Six-node triangles have been used yielding 12369 dofs. The mesh is refined near contact regions with a specified number of elements to properly discretize the contact width. Figure $3 \mathrm{~b}$ shows a zoom inside the contact region of the updated mesh after the application of a $0.6 \%$ tensile strain. Six three-node line elements have been used to discretize the corresponding contact width, resulting in 13 contact nodes and six load increments $(J=6)$.

Figure $4 \mathrm{a}, 4 \mathrm{~b}$ and $4 \mathrm{c}$ give the normalized plots of the axial force $F_{0}$, the twisting moment $M_{0}$ and the normal contact force $N_{0}$ as a function of $\epsilon$. Results are compared with Costello's analytical solution [22], briefly recalled in Appendix B. Numerical results are in good agreement with Costello's solutions, although the latter derives from a simplified beam model which does not account for contact. This is indeed consistent with the results of Ref. [23], where it has been found that the global static behavior of a strand is nearly insensitive to contact assumptions.

Figure 4d shows the normalized contact half-width $a$ as a function of the normal contact force computed from the FE model. Also shown is the theoretical solution obtained from Hertz theory for parallel cylinders, given by Eq. (6). A close agreement is found, which validates the static modeling of contact. This also shows that the effect of helical geometry is thus negligible on the contact width. Note that accounting for the helical geometry is yet necessary for the computation of $N_{0}$ (if parallel wires were considered, the contact force would remain equal to zero).

It must be empasized that the contact half width gets better approximated as it increases. This phenomenon is well-known in the FE analysis of contact mechanics [24]: as the load decreases, the contact width gets narrower and the number of contacting nodes in the FE model becomes insufficient to yield a correct approximation of contact.

The convergence of results has been checked by refining the mesh of Fig. 3. A $h$-refinement was used by dividing each triangle of the initial mesh into four triangles, generating four times more elements than the preceding mesh and 46893 dofs. Results are shown in Fig. 4. The contact width is slightly different with the refined mesh, but changes can be considered as small. The difference between both meshes is negligible for the maximum applied strain $\epsilon=0.6 \%$. As a consequence, the initial mesh (12369 dofs) can be considered 


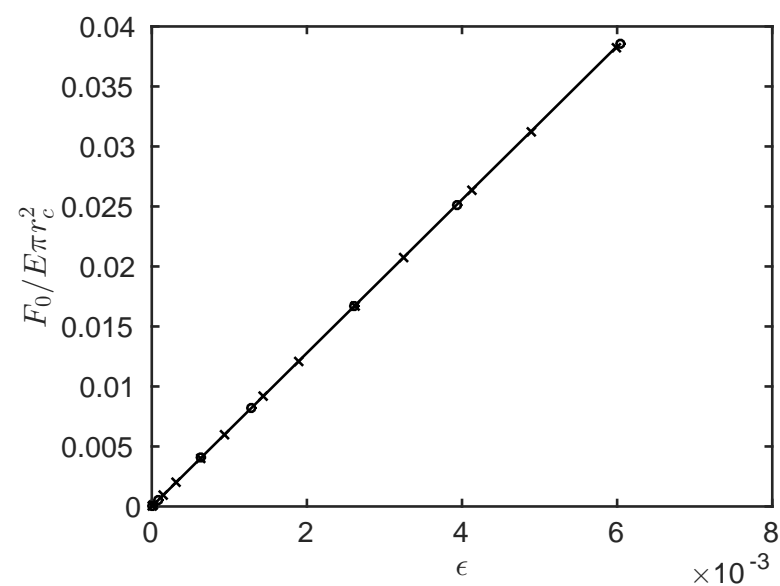

(a)

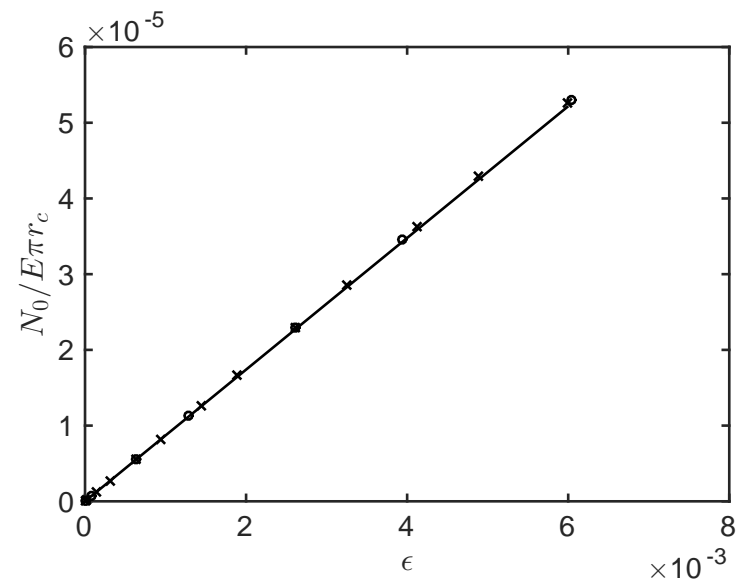

(c)

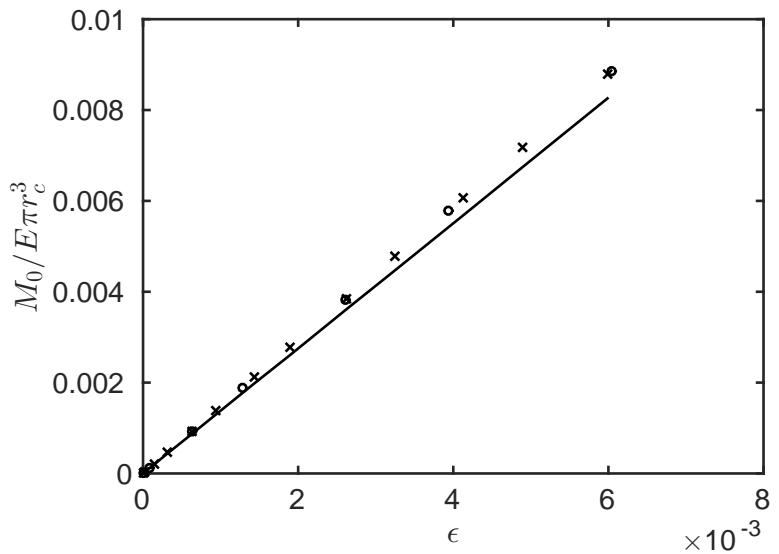

(b)

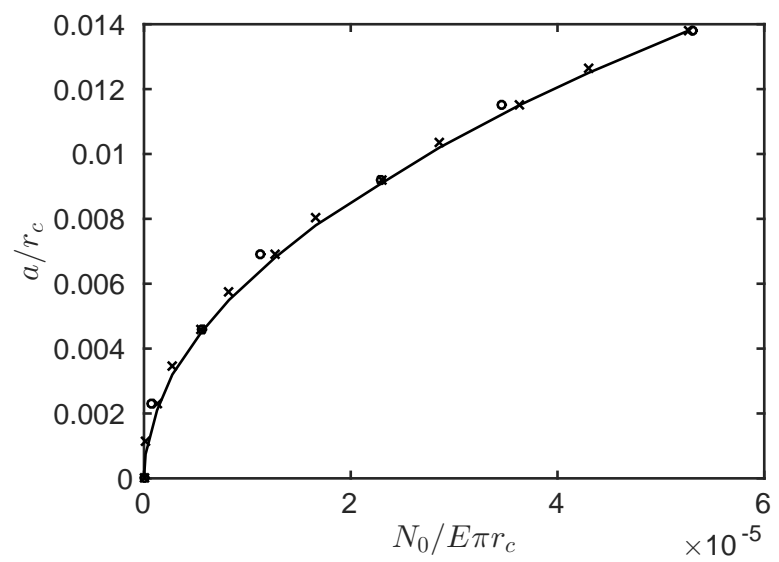

(d)

Figure 4: (a) Normalized axial force, (b) twisting moment, (c) normal contact force as a function of the applied strain $\epsilon$, and (d) contact half-width as a function of the normal contact force. Circles: numerical results with the initial mesh (12369 dofs), crosses: numerical results with refined mesh (46893 dofs), continuous lines: analytical solutions (Costello/Hertz). 
as sufficient for a proper contact modeling at $\epsilon=0.6 \%$.

It has to be noticed that the above results show that $N_{0}$ and then $a$ can be properly estimated thanks to the analytical formula (B.4) and (6). This allows to discretize the contact width with the desired number of elements prior to any FE computations.

As a side remark, a tensile strain $\epsilon=0.6 \%$ yields dimensional force $F_{0}=190.1 \mathrm{kN}$ and moment $M_{0}=$ 118.1 N.m, which roughly corresponds to the operational load of strands. These values are quite close to those obtained in Ref. [7] from a point-to-point contact approximation $\left(F_{0}=190.3 \mathrm{kN}\right.$ and $\left.M_{0}=118.1 \mathrm{~N} . \mathrm{m}\right)$, owing to the weak influence of contact assumptions on global statics as mentioned earlier.

As opposed to Hertz theory (frictionless), the above numerical results have been obtained from stick contact conditions. Though not shown for conciseness, numerical tests have also been performed in the slip case (displacement continuity along the normal direction only, the motion being free in the tangential direction owing to the frictionless assumption). No differences have been found with the results of Fig. 4 . Interwire slip is actually negligible for the type of loads considered in this paper, i.e. tensile strain with zero rotational strain. This confirms that shear contact forces and friction can be neglected in the present static analysis.

\section{Dispersion curves}

Once the static prestress state has been determined, the propagation of guided waves can be analyzed. The dynamic analysis is performed by solving the SAFE eigenproblem, corresponding to small amplitude wave modes superimposed on the prestress state. Our attention is restricted to propagating modes $(k \in \mathbb{R})$. Setting $k$ to fixed values, the SAFE system (1) is a linear eigenproblem for the eigenvalue $\omega$. Dispersion curves can then be plotted as a function of frequency. In this section, curves will be presented for the energy velocity, which can be post-processed from SAFE matrices as follows [8]:

$$
v_{e}=\frac{2 \omega \operatorname{Im}\left\{\mathbf{U}^{\mathrm{T} *}\left(\mathbf{K}_{2 \sigma}^{\mathrm{T}}+\mathrm{i} k \mathbf{K}_{3 \sigma}\right) \mathbf{U}\right\}}{\operatorname{Re}\left\{\mathbf{U}^{\mathrm{T} *}\left(\mathbf{K}_{1 \sigma}+\omega^{2} \mathbf{M}+\mathrm{i} k\left(\mathbf{K}_{2 \sigma}-\mathbf{K}_{2 \sigma}^{\mathrm{T}}\right)+k^{2} \mathbf{K}_{3 \sigma}\right) \mathbf{U}\right\}}
$$

where the superscripts $T$ and $*$ denote the matrix transpose and the complex conjuguate respectively.

The main goal of this section is to understand the origin of the notch frequency phenomenon occurring in seven-wire strands. In that purpose, the combined effects of prestress, contact, Poisson coefficient and lay angle are investigated. The convergence of results is briefly discussed. Unless otherwise noted, stick contact conditions will be assumed. The influence of slip will be briefly examined to conclude this section.

\subsection{Preliminary results}

Figure 5 shows the normalized energy velocity dispersion curves for the seven-wire strand subjected to an axial strain of $\epsilon=0.6 \%$. The normalized frequency is given by $\omega r_{c} / c_{s}$, with $c_{s}=\sqrt{E / 2 \rho(1+\nu)}$ denoting the shear wave velocity. With the same dimensional parameters as given by Sec. 3.3, the dimensional frequency here ranges from 0 to $390 \mathrm{kHz}$.

Many propagating modes are found. Conversely for a single wire disconnected from the others, it should be recalled that only three modes are propagating in the frequency range $\omega r_{c} / c_{s} \in[0 ; 1.8]$ : the first longitudinal, torsional and flexural modes, denoted as $\mathrm{L}(0,1), \mathrm{T}(0,1)$ and $\mathrm{F}(1,1)$ respectively (see Ref. [6] for instance). The presence of many propagating modes in Fig. 5 is indeed intrinsic to the multi-wire nature of the waveguide.

Of particular interest in this section is the first longitudinal mode. Instead of a single continuous curve, this modes turns out to split in two curves in a seven-wire strand, denoted as $\mathrm{L}(0,1)$ a and $\mathrm{L}(0,1) \mathrm{b}$ in Fig. 5 . This split indeed corresponds to a curve veering phenomenon [6], centered around the frequency $\omega r_{c} / c_{s}=0.43$, which coincides with the so-called notch frequency of the literature. The origin of this phenomenon is investigated in the remainder. 


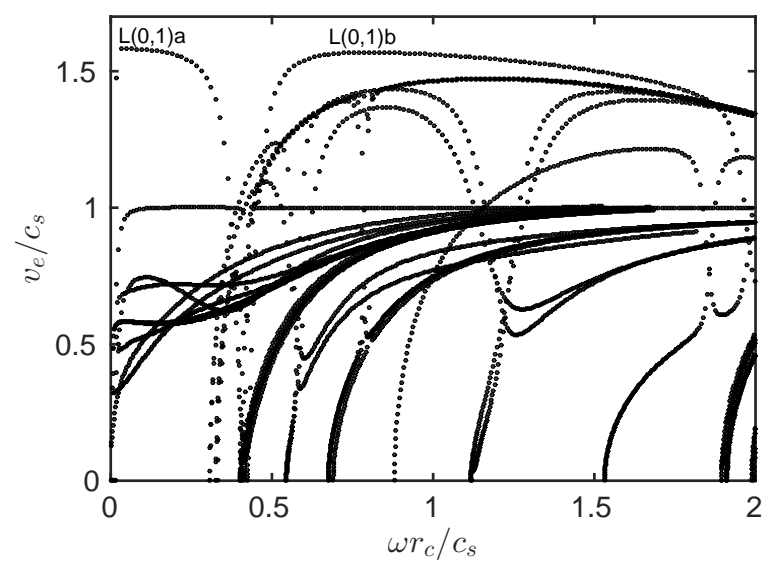

Figure 5: Dispersion curves of the loaded seven-wire strand $(\epsilon=0.6 \%)$.

\subsection{Effect of axial load on the notch frequency and convergence}

Figure 6 a compares the curve veering of the $\mathrm{L}(0,1)$ mode computed in the unloaded case $(\epsilon=0 \%)$ and in the loaded case $(\epsilon=0.6 \%)$. The normalized curve veering frequency, which can be defined as the cut-off of the $\mathrm{L}(0,1) \mathrm{b}$ mode, increases from 0.32 to 0.42 , yielding dimensional values of $62 \mathrm{~Hz}$ and $82 \mathrm{~Hz}$ without and with tensile strain respectively. Both values are consistent with the notch frequency observed in experiments $[1,2]$.

As a numerical test, Fig. 6b shows the curve veering occurring in an artificial configuration, unloaded but with the same contact width as for the $\epsilon=0.6 \%$ case (i.e. 13 interwire contact node pairs instead of one). Results are almost identical to those of Fig. 6a, which proves that the increase of the notch frequency is mainly explained by the increase of the interwire contact width rather than the prestress $\boldsymbol{\sigma}_{0}$ itself.

Owing to this strong influence of contact width, the accuracy of numerical results has to be discussed. Figure 6 shows the results computed with the refined mesh of the previous section (46893 dofs). It can be observed that changes are negligible for $\epsilon=0.6 \%$, as opposed to the unloaded case for which a slight shift is found towards low frequency. For $\epsilon=0.6 \%$, it can be concluded that a reasonable accuracy is achieved with the inital mesh. This confirms that 13 contact points are sufficient to discretize the contact width.

As far as the unloaded case is concerned, a single point contact is used in the simulations. Such a configuration is somehow purely theoretical. A single contact point can indeed be viewed as a singular point. In contact mechanics, it is well known that a minimum number of elements is required to discretize a given contact width (see Refs. [24, 25] for instance), which means that the FE mesh must be refined in the contact region as the contact width decreases. To the limiting case of single contact point (i.e. zero width), the size of elements should hence tend to zero. Therefore, convergence can hardly be achieved for the theoretically unloaded case.

However in practice, the computed veering frequency does not vary much between both meshes $(0.31$ with the refined mesh against 0.32 with the initial one) and the initial mesh will be retained in the remainder of this paper for simplicity.

\subsection{Influence of Poisson coefficient and lay angle on the notch frequency}

The influence of two key parameters, the Poisson coefficient and the helix lay angle, is now studied. One is interested in highlighting the origin of the veering phenomenon. As it still occurs in the unloaded case, such a phenomenon turns out to be intrinsic to the geometry. For simplicity, only the unloaded case is considered in this subsection. This allows to reduce by one the number of parameters ( $\epsilon$ is set to zero) and avoids the combined effects of contact and lay angle under prestress. Otherwise for a given non zero load, the contact width would vary with the lay angle (for instance in the limiting case of $\phi=0^{\circ}$, i.e. straight peripheral wires, the contact remains single point for any tensile strain values). 


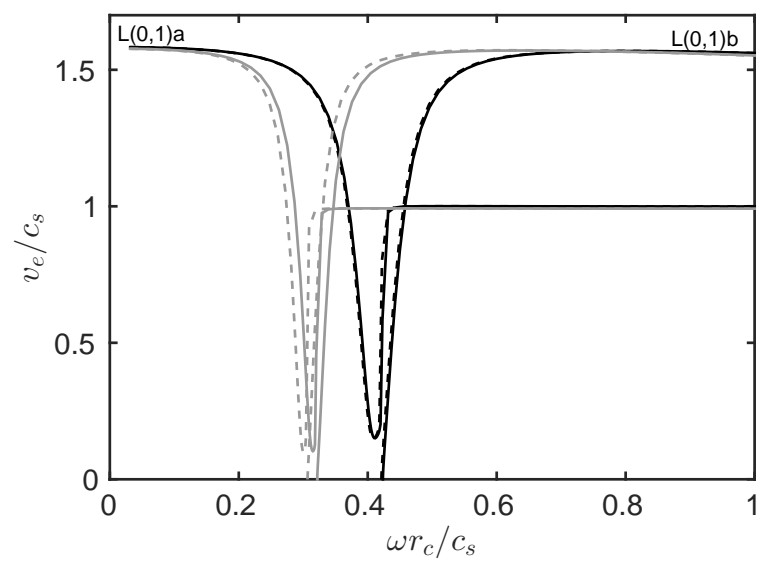

(a)

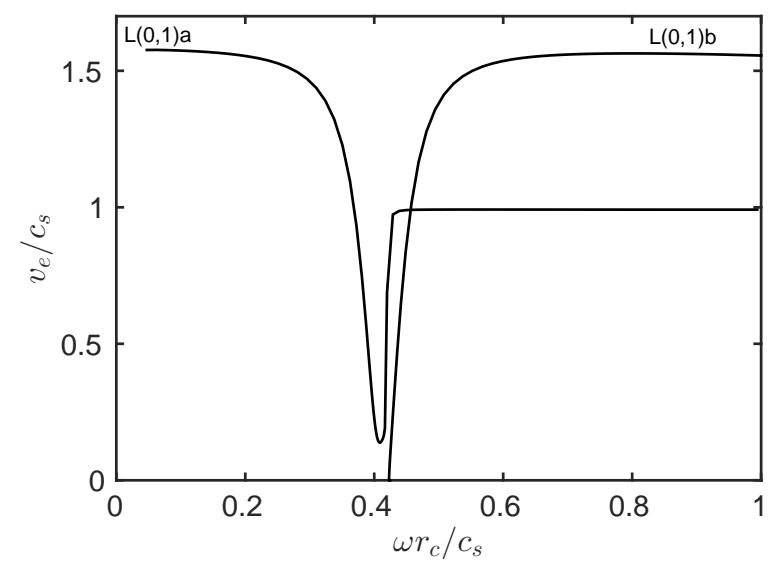

(b)

Figure 6: (a) Curve veering for the unloaded strand (gray, $\epsilon=0 \%$ ) and for the loaded strand (black, $\epsilon=0.6 \%$ ). Continuous lines: results with the initial mesh (12369 dofs), dashed lines: results with the refined mesh (46893 dofs). (b) Artificial configuration, unloaded but with the same contact width as for $\epsilon=0.6 \%$.

Figure 7a exhibits the curve veering results computed for $\phi=0^{\circ}$. Although narrowed compared to Fig. 6 , the velocity drop associated with the veering frequency still occurs at the same frequency. Figure $7 \mathrm{~b}$ shows the results obtained for $\nu=0$ and $\phi=7.9^{\circ}$. The veering phenomenon appears to be significantly narrowed and is shifted to lower frequency (note also that the energy velocity decreases). Figure $7 \mathrm{~b}$ also plots the curve when both the Poisson coefficient and the helix lay angle are set to zero. In this case, the veering phenomenon completely disappears.

These results shows that both the Poisson effect and the helical geometry are responsible for the $\mathrm{L}(0,1)$ curve veering in seven-wire strands. This suggests that the origin of this phenomenon lies in the radial motion of longitudinal modes in wires. On the one hand, this radial motion is caused by Poisson effects (as it typically occurs in straight waveguides). On the other hand, the helical geometry of peripheral wires yields additional radial motion: in a peripheral wire, the longitudinal mode is mainly oriented along the helical axis of the wire so that a radial component is always generated in the cross-section plane (even for $\nu=0)$.

This explanation is confirmed by Fig. 8, showing the radial displacement of $\mathrm{L}(0,1)$ a and $\mathrm{L}(0,1) \mathrm{b}$ modes at one contact point. The modal displacement has been normalized by the mass matrix M. The radial displacement exhibits a sharp peak around the veering frequency. This result is consistent with the mode shapes found in Ref. [6], exhibiting a significant motion in the radial direction at the notch frequency.

As a side remark, it should be mentioned that curve veering may also occur for other modes involving significant radial expansion. In the numerical dispersion curves of Fig. 5, one can observe some sharp velocity drops, occurring for different modes and corresponding to veering phenomena also. Experimental studies may yet be needed to confirm their existence.

\subsection{Uncoupled blocked dispersion curves}

The following question arises: is the curve veering phenomenon predictable from the dispersion curves of uncoupled wires? Following the analysis of Ref. [26], curve veering phenomena in weakly coupled systems usually result from the intersection in the $(k, \omega)$ plane of the dispersion curves of the uncoupled system.

For a multi-wire strand, there are two ways of considering the uncoupled system: wires can be disconnected (zero interwire force) or blocked (zero interwire displacement). As far as the uncoupled disconnected case is considered, the dispersion curves are well-known as they roughly correspond to the PocchammerChree solution of a free cylinder [27]. However, the displacement peak observed in Fig. 8 for a strand does 


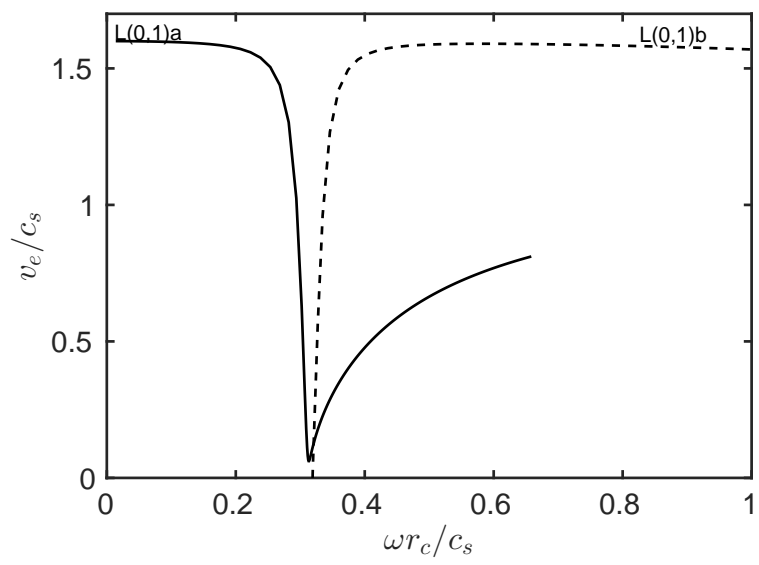

(a)

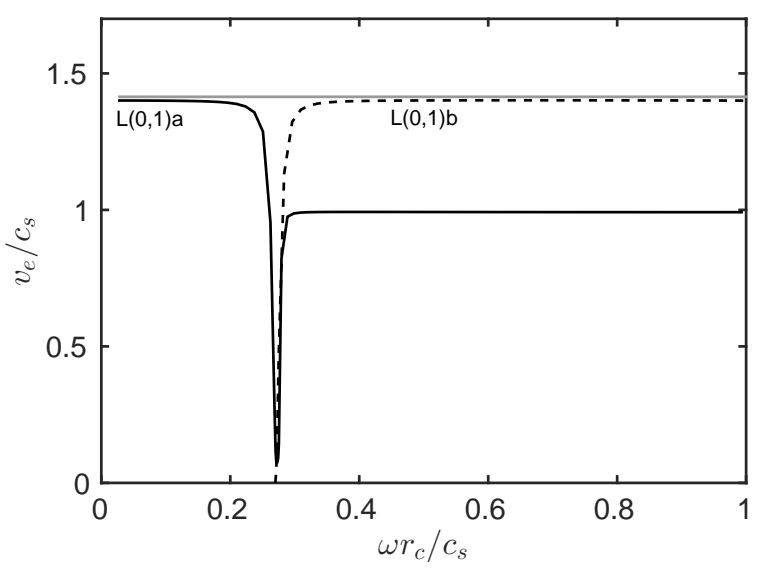

(b)

Figure 7: (a) Curve veering for $(\nu, \phi)=\left(0.28,0^{\circ}\right)$, (b) for $(\nu, \phi)=\left(0,7.9^{\circ}\right)$, gray line: results for $(\nu, \phi)=\left(0,0^{\circ}\right)$. Continuous line: $\mathrm{L}(0,1)$ a mode, dashed line: $\mathrm{L}(0,1) \mathrm{b}$ mode. The strand is unloaded $(\epsilon=0)$.

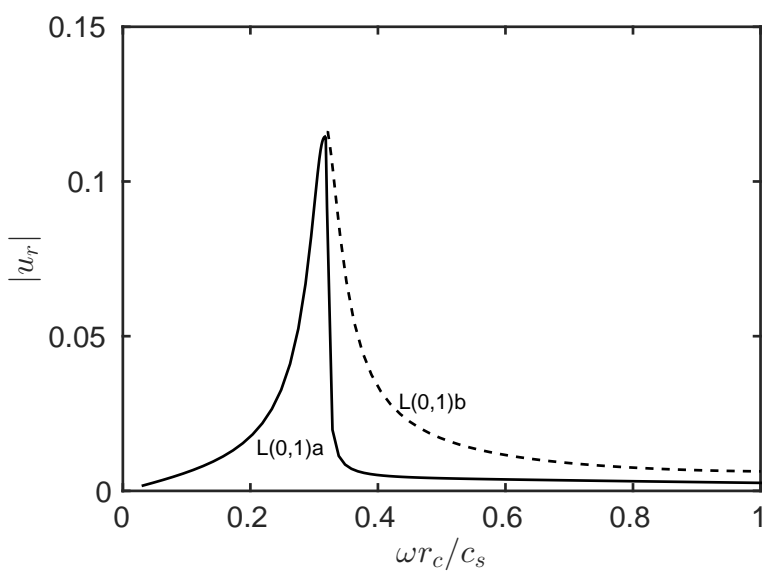

Figure 8: Normalized radial displacement at contact point of $\mathrm{L}(0,1)$ a mode (continuous line) and $\mathrm{L}(0,1) \mathrm{b}$ mode (dashed line) for: $\nu=0.28, \phi=7.9^{\circ}, \epsilon=0$. 
not occur in a free cylinder. Furthermore, it has been checked that no intersection occurs between the dispersion curves of the central wire and those of a peripheral wire (results not shown).

As for the uncoupled-blocked system, the interwire displacement can be blocked in one, two or three directions. Owing to the previous results, the radial direction appears to be the most critical and is set to zero. Figure 9a shows the energy velocity dispersion curves of longitudinal-like modes for the uncoupled central and peripheral wires, radially blocked at interwire contact regions (only one node). A similar curve veering phenomenon is found in the uncoupled blocked peripheral wire, although the veering phenomenon occurs at a slightly lower frequency in the fully coupled strand, which can be attributed to the elasticity of the central wire. Somewhat surprisingly, note that the dispersion curves of the uncoupled blocked central wire do not suffer from any veering and look like the $L(0,1)$ mode of a free cylinder (the central wire behavior is indeed basically different as six contact regions are constrained, so that a rotational symmetry is preserved).

Also shown in Fig. 9a are the results obtained with a zero radial displacement artificially enforced at the 13 contact nodes corresponding to the $\epsilon=0.6 \%$ contact width (computations are still performed without load, i.e. $\epsilon=0$ ). Similarly to the fully coupled strand, the curve veering increases with the number of constrained nodes.

The origin of the veering phenomenon can indeed be explained by the dispersion curves in the $(k, \omega)$ plane of the uncoupled radially blocked peripheral wire, given in Fig. 9b. Like for a free cylinder, four propagating modes can be found in the frequency range. Yet in a free cylinder, it is well-known that longitudinal, torsional and flexural modes are uncoupled from each other [27]. Blocking the displacement at one or a few points on the cross-section boundary completely breaks the circular symetry so that modes get coupled.

The plot in the $(k, \omega)$ plane allows to clearly view the veering phenomenon: the branches labeled as $\mathrm{L}(0,1) \mathrm{a}$ and $\mathrm{L}(0,1) \mathrm{b}$ suddenly veer away from each other near the frequency $\omega r_{c} / c_{s}=0.6$ instead of crossing. Note that in the $\left(v_{e}, \omega\right)$ plane, both branches are allowed to cross each other. One recalls that the energy velocity $v_{e}$ is equal to the group velocity $v_{g}$ (provided that the waveguide is lossless). Since $v_{g}=\mathrm{d} \omega / \mathrm{d} k, v_{e}$ gives a measure of the slopes, which rapidly change near a veering frequency but may intersect.

As shown in Fig. 9b in the low frequency range, the longitudinal-like mode curve, labeled as L(0,1)a, veers around the wavenumber $k r_{c}=0.4$ and then adopts the motion of a flexural-like mode. The curve veers once again around $k r_{c}=0.6$, to switch to a torsional motion (this veering is extremely narrow and curves seems to intersect in the figure).

Of particular interest is the curve of the flexural motion cut-off at the frequency 0.6. It can be observed that this curve quickly veers to adopt a longitudinal motion at higher frequency, labeled as $\mathrm{L}(0,1) \mathrm{b}$.

It can be concluded that the origin of the veering phenomenon lies in the coupling between two modes, one of longitudinal type and the other of flexural type, which results from the break of the circular symmetry caused by the radial displacement constraint applied on the interwire boundary of peripheral wires.

\subsection{Discussion on slip contact conditions}

Previous results have been obtained with stick conditions. Assuming stick contact conditions somehow means that peripheral wires can roll without sliding on the central wire (analogy with wheels [19]). It amounts to say that tangential forces induced by waves remain sufficiently small. From a theoretical point of view, slip may occur and yield friction when the tangential forces exceed a certain limit.

Figure 10 presents the dispersion curves computed for $\epsilon=0.6 \%$ in the frictionless case (slip contact conditions). The motion of each wire is then free along the tangential directions inside the contact region (but the normal displacement continuity is still applied to ensure non-penetration). Unlike for statics, the type of contact conditions can strongly influence the wave modes of the structure (compare Fig. 10 with Fig. 5).

A curve veering still occurs around the notch frequency $\left(\omega r_{c} / c_{s}=0.42\right)$. However, some other modes of longitudinal type do not suffer from veering around the notch frequency. This feature does not seem to be compatible with experimental results. The longitudinal nature of these unveering modes has been checked from a visual inspection of mode shapes. A significant peripheral motion has been found in these modes, which should have hence been detected from the magnetostrictive measurements reported in Refs. $[1,2]$. It 


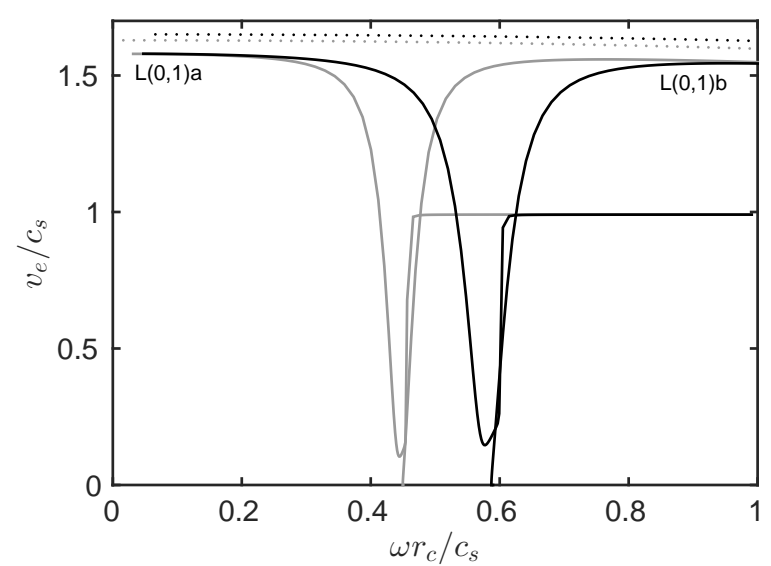

(a)

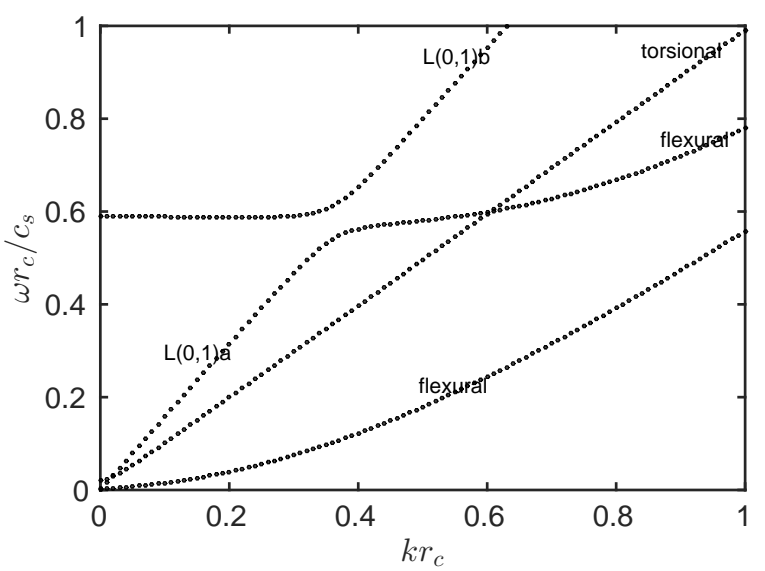

(b)

Figure 9: (a) Curve veering for an unloaded uncoupled peripheral wire having a radial displacement blocked along its interwire contact boundary for a contact width corresponding to $\epsilon=0$ (only one constrained node, gray) and to $\epsilon=0.6 \%$ ( 13 constrained nodes, black). Dotted lines are for the central wire (uncoupled radially blocked). (b) Dispersion curves in the ( $k, \omega)$ plane for the uncoupled blocked peripheral wire (13 constraints).

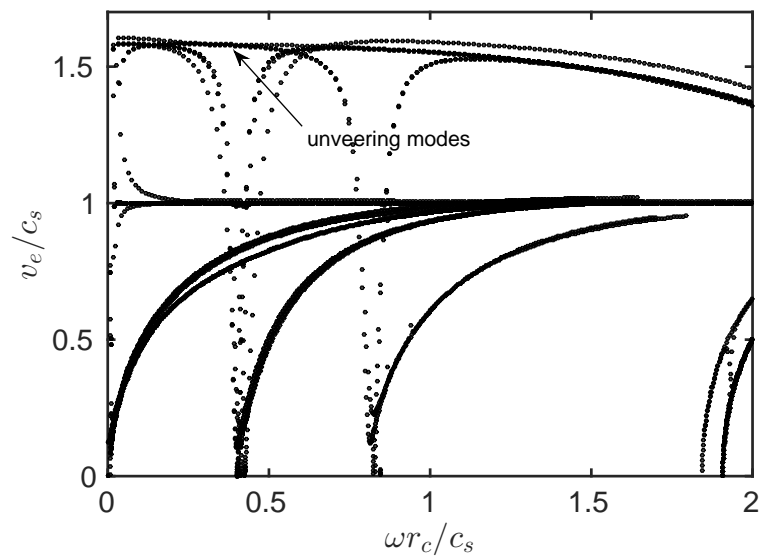

Figure 10: Dispersion curves of the loaded seven-wire strand with slip contact conditions $(\epsilon=0.6 \%)$. 
can hence be inferred that slip conditions are less realistic for wave propagation of longitudinal-like modes in seven-wire strands.

\section{Conclusion}

This paper highlights how tensile loads can significantly increase the notch frequency of longitudinal modes inside seven-wire strands. Rather than prestress itself, the main mechanism lies in the increase of interwire contact width (increase of contact node pairs in the numerical model).

The static computation of the prestress state has been validated based on Costello's solution for multiwire ropes (neglecting contact) as well as based on Hertz's contact solution (for parallel cylinders). Numerical results are shown to be accurate provided that a sufficient number of elements is used to discretized the interwire contact width. In the unloaded case, the model hardly converges because the contact width tends to zero, but differences remains reasonably small as far as the notch frequency is concerned.

Although stick contact conditions have been considered throughout the paper, the influence of slip conditions has been briefly discussed: although negligible on statics, slip conditions strongly affects the dispersion curves of longitudinal-like modes, which appear to be incompatible with experimental observations.

The dispersion curves of the seven-wire strand have been computed by varying the Poisson coefficient and the helix lay angle. Results have proved the importance of the radial motion of wires in the curve veering of longitudinal modes, and in the limiting case of vanishing Poisson coefficient and lay angle, the veering phenomenon completely disappears.

Finally, it has been demonstrated that a curve veering of longitudinal modes can similarly be found in a single wire, uncoupled from the others, when its radial displacement is blocked in a localized zone corresponding to the interwire contact region. Such a localised boundary condition completely breaks the circular symmetry of the wire cross-section, yielding coupling between longitudinal, flexural and torsional modes, and resulting in curve veering phenomena. Therefore, it can be deduced that the origin of the curve veering of longitudinal modes inside seven-wire strands lies in the radial displacement constraint imposed on peripheral wires by the central one in the interwire contact regions.

\section{Appendix A. Expressions of operators}

The matrix $\Sigma_{0}$ is defined by:

$$
\boldsymbol{\Sigma}_{0}=\left[\begin{array}{ccc}
\boldsymbol{\sigma}_{0} & \mathbf{0} & \mathbf{0} \\
\mathbf{0} & \boldsymbol{\sigma}_{0} & \mathbf{0} \\
\mathbf{0} & \mathbf{0} & \boldsymbol{\sigma}_{0}
\end{array}\right]
$$

The operators $\mathbf{L}_{x y}, \mathbf{L}_{z}, \mathbf{G}_{x y}, \mathbf{G}_{z}$ are:

$$
\begin{aligned}
\mathbf{L}_{x y}= & {\left[\begin{array}{ccc}
\partial / \partial x & 0 & 0 \\
0 & \partial / \partial y & 0 \\
0 & 0 & \Lambda \\
\partial / \partial y & \partial / \partial x & 0 \\
\Lambda & -\tau_{0} & \partial / \partial x \\
\tau_{0} & \Lambda & \partial / \partial y
\end{array}\right], \mathbf{L}_{z}=\left[\begin{array}{lll}
0 & 0 & 0 \\
0 & 0 & 0 \\
0 & 0 & 1 \\
0 & 0 & 0 \\
1 & 0 & 0 \\
0 & 1 & 0
\end{array}\right], } \\
\mathbf{G}_{x y}= & {\left[\begin{array}{ccc}
\partial / \partial x & 0 & 0 \\
\partial / \partial y & 0 & 0 \\
\Lambda & -\tau_{0} & 0 \\
0 & \partial / \partial x & 0 \\
0 & \partial / \partial y & 0 \\
\tau_{0} & \Lambda & 0 \\
0 & 0 & \partial / \partial x \\
0 & 0 & \partial / \partial y \\
0 & 0 & \Lambda
\end{array}\right], \mathbf{G}_{z}=\left[\begin{array}{lll}
0 & 0 & 0 \\
0 & 0 & 0 \\
1 & 0 & 0 \\
0 & 0 & 0 \\
0 & 0 & 0 \\
0 & 1 & 0 \\
0 & 0 & 0 \\
0 & 0 & 0 \\
0 & 0 & 1
\end{array}\right] }
\end{aligned}
$$

where $\Lambda=\tau_{0}(y \partial / \partial x-x \partial / \partial y)$. 


\section{Appendix B. Costello's formula}

From Ref. [22], the rotational strain $\beta$ of a peripheral wire can be expressed as:

$$
\beta=\left\{(1+\nu) \tan \phi+\nu r_{c} / r_{p}\right\} \epsilon+\left\{1+(1+\nu) \tan ^{2} \phi\right\} \Delta \phi
$$

where $\epsilon$ is the prescribed axial strain applied on the strand and $\Delta \phi$ denotes the lay angle change. The total axial force $F_{0}$, the total twisting moment $M_{0}$ acting on the strand are given by:

$$
\begin{array}{r}
F_{0}=E r_{c}^{2} \pi \epsilon+6 E r_{p}^{2}(t \cos \phi+n \sin \phi), \\
M_{0}=E r_{c}^{3} \frac{\pi r_{c}}{4(1+\nu) r_{p}} \beta+6 E r_{p}^{3}\left(\frac{r_{c}+r_{p}}{r_{p}}(t \sin \phi-n \cos \phi)+h \cos \phi+g \sin \phi\right)
\end{array}
$$

with notations:

$$
\begin{array}{r}
g=\frac{\pi}{4} r_{p} \Delta \kappa, \quad h=\frac{\pi}{4(1+\nu)} r_{p} \Delta \tau, \quad t=\pi(\epsilon+\Delta \phi \tan \phi), \quad n=\kappa_{p} r_{p} h-\tau_{p} r_{p} g, \\
\Delta \kappa=2 \tau_{p} \Delta \phi+\nu\left(\epsilon+r_{p} \Delta \phi \tan \phi /\left(r_{c}+r_{p}\right)\right) \kappa_{p}, \\
\Delta \tau=-\left(2 \kappa_{p}-1 /\left(r_{c}+r_{p}\right)\right) \Delta \phi+\nu\left(\epsilon+r_{p} \Delta \phi \tan \phi /\left(r_{c}+r_{p}\right)\right) \tau_{p}
\end{array}
$$

where $\tau_{p}=\sin \phi \cos \phi /\left(r_{c}+r_{p}\right)$ and $\kappa_{p}=\sin ^{2} \phi /\left(r_{c}+r_{p}\right)$ denotes the torsion and the curvature, in the undeformed configuration, of the helical axis of peripheral wires respectively. The normal contact force per unit length acting along one peripheral wire is:

$$
N_{0}=E r_{p}^{2}\left(n \tau_{p}-t \kappa_{p}\right)
$$

In this paper, the strand is not allowed to rotate, so that $\beta=0$ and the lay angle change $\Delta \phi$ can be readily obtained from the linear solution of Eq. (B.1).

\section{References}

[1] H. Kwun, K. A. Bartels, J. J. Hanley, Effects of tensile loading on the properties of elastic-wave propagation in a strand, Journal of the Acoustical Society of America 103 (1998) 3370-3375.

[2] L. Laguerre, M. Brissaud, J. C. Aime, Low-frequency ultrasound reflectometry device based on magnetoelastic transducers for the non destructive evaluation of steel rods and cables, Bulletin des Laboratoires des Ponts et Chaussées 239 (2002) $7-27$.

[3] P. Rizzo, F. L. di Scalea, Load measurement and health monitoring in cable stays via guided wave magnetostrictive ultrasonics, Materials Evaluation 62 (2004) 1057-1065.

[4] F. Treyssède, Numerical investigation of elastic modes of propagation in helical waveguides, Journal of the Acoustical Society of America 121 (2007) 3398-3408.

[5] F. Treyssède, Elastic waves in helical waveguides, Wave Motion 45 (2008) 457-470.

[6] F. Treyssède, L. Laguerre, Investigation of elastic modes propagating in multi-wire helical waveguides, Journal of Sound and Vibration 329 (2010) 1702-1716.

[7] A. Frikha, P. Cartraud, F. Treyssède, Mechanical modeling of helical structures accounting for translational invariance. part 1: Static behavior, International Journal of Solids and Structure 50 (2013) 1373-1382.

[8] F. Treyssède, A. Frikha, P. Cartraud, Mechanical modeling of helical structures accounting for translational invariance. part 2: Guided wave propagation under axial loads, International Journal of Solids and Structure 50 (2013) 1383-1393.

[9] N. C. Perkins, C. D. Mote, Comments on curve veering in eigenvalue problems, Journal of Sound and Vibration 106 (1986) 451-463.

[10] C. Pierre, Mode localization and eigenvalue loci veering in disordered structures, Journal of Sound and Vibration 126 (1988) 485-502.

[11] L. Gavric, Computation of propagative waves in free rail using a finite element technique, Journal of Sound and Vibration 185 (1995) 531-543.

[12] I. Bartoli, A. Marzani, F. L. di Scalea, E. Viola, Modeling wave propagation in damped waveguides of arbitrary crosssection, Journal of Sound and Vibration 295 (2006) 685-707.

[13] S. Finnveden, M. Fraggstedt, Waveguide finite elements for curved structures, Journal of Sound and Vibration 312 (2008) 644-671.

[14] P. Sabiniarz, W. Kropp, A waveguide finite element aided analysis of the wave field on a stationary tyre, not in contact with the ground, Journal of Sound and Vibration 329 (2010) 3041-3064. 
[15] O. Onipede, S. B. Dong, Propagating waves and end modes in pretwisted beams, Journal of Sound and Vibration 195 (1996) 313-330.

[16] F. Treyssède, Mode propagation in curved waveguides and scattering by inhomogeneities: application to the elastodynamics of helical structures, Journal of the Acoustical Society of America 129 (2011) 1857-1868.

[17] K. J. Bathe, Finite Element Procedures, Prentice Hall, Englewood Cliffs, New Jersey, 1996.

[18] K. L. Johnson, Contact Mechanics, University Press, Cambridge, 1985.

[19] P. Wriggers, Computational Contact Mechanics, Springer-Verlag, Berlin, 2006.

[20] A. Nawrocki, M. Labrosse, A finite element model for simple straight wire rope strands, Computers \& Structures 77 (2000) 345-359.

[21] C. Geuzaine, J.-F. Remacle, Gmsh: a three-dimensional finite element mesh generator with built-in pre- and postprocessing facilities, International Journal for Numerical Methods in Engineering 79 (11) (2009) 1309-1331.

[22] G. A. Costello, Theory of wire ropes, Springer-Verlag, New York, 1990.

[23] S. R. Ghoreishi, T. Messager, P. Cartraud, P. Davies, Validity and limitations of linear analytical models for steel wire strands under axial loading, using a 3D FE model, International Journal of Mechanical Sciences 49 (2007) $1251-1261$.

[24] S. K. Chan, I. S. Tuba, A finite element method for contact problems of solid bodies - Part I. Theory and validation, International Journal of Mechanical Sciences 13 (1971) 615-625.

[25] N. Chandrasekaran, W. E. Haisler, R. E. Goforth, Finite element analysis of Hertz contact problem with friction, Finite Elements in Analysis and Design 3 (1987) 39-56.

[26] B. R. Mace, E. Manconi, Wave motion and dispersion phenomena: Veering, locking and strong coupling effects, Journal of the Acoustical Society of America 131 (2012) 1015-1028.

[27] J. Zemanek, An experimental and theoretical investigation of elastic wave propagation in a cylinder, Journal of the Acoustical Society of America 51 (1972) 265-283. 